\title{
The role of emotion in mental maps
}

\author{
Mariam Gambashidze, Juliane Cron* \\ Technical University of Munich, mariam.gambashidze@tum.de, juliane.cron@tum.de \\ * Corresponding author
}

Keywords: mental map, emotion, places, 'image' of a city

\begin{abstract}
:
Mental mapping is "visual speaking through places what words alone cannot articulate"; it can be seen as a method of exploring subjective meanings of space, conveying knowledge and experiencing it in a different form (Gieseking, 2013). Mental mapping intersects many disciplines such as cartography, psychology, environment-behaviour studies and its history dates back to the beginning of the $20^{\text {th }}$ century. However, the watershed moment for research into environmental cognition followed Kevin Lynch's (1960) book 'The Image of the City' (Downs \& Meyer, 1978) with a focus on designing a legible city (Gieseking, 2013). In his work, Lynch questioned how inhabitants perceive their environment, what elements form a place, and how cities can be organized in a better way (Lynch, 1960). The applied method of mental mapping has expanded over time; still, it remains a question of how we can use it as a research method (Gieseking, 2013) in cartography and what benefits this provides.
\end{abstract}

Besides mental mapping, the role of emotion also falls outside the main scope of traditional cartographic research. Historically, cartographic research has mainly focused on representing visible and tangible phenomena (Griffin \& Mcquoid, 2012; Panek \& Benediktsson, 2017). Whereas the study of the emotional component and human experiences in human-environment relationships is rarely considered and does not have a long history (Griffin \& Mcquoid, 2012; Pearce, 2008).

Places and emotions are related to each other and these relationships are complex (Caquard \& Griffin, 2018). Places can evoke various emotional responses which can be translated into mental images. These responses are studied in emotional cartography by different means such as biometric measurements (Nold, 2009), user-generated content (Hauthal \& Burghardt, 2019), and surveys (Huang et al., 2014). It is not well understood, however, how our emotions influence our attitude and behaviour towards a place. Furthermore, if we consider that mental images are not static fluid and dynamic, then we may ask how they change as our knowledge and experience about the place grow.

In our research, we explored the aspects which shape our mental image of the place as it is perceived and experienced by individuals. We applied a synthesis of existing methodologies and suggest a new framework to analyze and interpret mental maps. Within this study mental maps are understood as graphical representations of a space (Dernat et al., 2016; Reuchamps et al., 2013) that hold a cognitive and an emotional component (Milgram \& Jodelet, 1976). We aim to explain the connection between emotion and mental maps. Furthermore, our study intends to compare the differences between the mental images that are drawn before visiting and after exploring and living in that place. The focus of the research is exploring the personal understanding of the place which is affected by the cognitive memory (i.e. patterns stored in memory through experience and repetition), existing knowledge, emotions, associations and experiences. The visual language of mental maps and emotional attachments to places has been studied based on mental maps, questionnaires and interviews. A combination of visual (i.e. mental maps hand-drawn by participants) and verbal language (discourses on the drawings) have been used to give different insights to understand the mental image of a place.

Vienna was chosen as a case study site due to its highly image-able character. This character is comprised of elements such as recognizable landmarks, vivid urban identity, and memorable features created by its history, inhabitants, and visitors. All these elements have perceptual importance and evoke a strong and easily identifiable image of the city enriched with the remembered experiences of and emotional attachments to Vienna. The participants of the user studies were a cohort of students of the International Master program in Cartography (www.cartographymaster.eu), who have not lived in Vienna before and moved there for their studies.

The first user study was completed via a video conference before the participants moved to Vienna. The study consisted of questionnaires, a narrated presentation about the 'Vienna city walk' (a virtual tour), and a mental mapping activity. The second user study was conducted on-site after the participants of the first user study had moved to and been living in Vienna for over three months. In this study, different methods were combined again, such as 'Vienna city walk'(on-site), mental mapping, questionnaire, and interviews (Figure 1). The outcome of both user studies were 52 hand-drawn mental maps, as well as quantitative and qualitative data from the above-mentioned questionaires and interviews. 


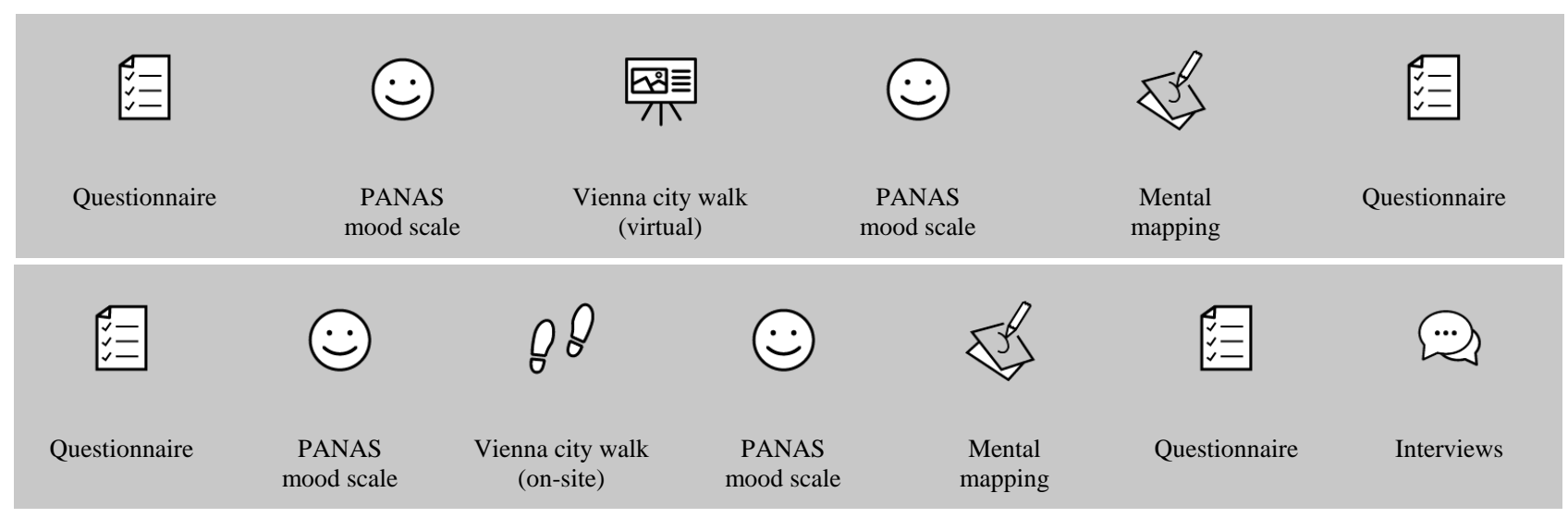

Figure 1. Structure of the first (above) and second user study (below).

The mental maps were analysed by categorising their composite elements into the groups such as landmarks \& buildings, art \& culture, urban environment, parks \& nature, river \& surroundings. Furthermore, the resulted mental maps can be assembled in the following categories: (1) consistent mental images, (2) altered mental images, (3) 'Vienna city walk' depicting mental images, and (4) particular mental images. The user studies have shown that several landmarks have perceptual importance and evoke a strong and easily identifiable image of the city enriched with the remembered experiences of and emotional attachments to Vienna. According to our analysis, the participants' emotional attachments to the places grow as they become acquainted with the places. Having similar experiences, however, does not ensure a similar visual outcome; mental maps may significantly differ from person to person as they are affected by the associations, existing knowledge, and personal experiences. Yet using mapping, as well as qualitative and quantitative methods of data collection, cannot assure that emotions can be easily tracked and described in mental maps and narrations.

\section{References}

Caquard, S., \& Griffin, A. (2018). Mapping Emotional Cartography. Cartographic Perspectives, 91, 4-16-4-16.

Dernat, S., Johany, F., \& Lardon, S. (2016). Identifying choremes in mental maps to better understand socio-spatial representations. Cybergeo, 2016. https://doi.org/10.4000/cybergeo.27867.

Downs, R. M., \& Meyer, J. T. (1978). Geography and the Mind: An Exploration of Perceptual Geography. American Behavioral Scientist, 22(1), 59-77. https://doi.org/10.1177/000276427802200104.

Gieseking, J. J. (2013). Where We Go From Here: The Mental Sketch Mapping Method and Its Analytic Components. Qualitative Inquiry, 19(9), 712-724. https://doi.org/10.1177/1077800413500926.

Griffin, A., \& Mcquoid, J. (2012). At the intersection of maps and emotion: The challenge of spatially representing experience. Kartographische Nachrichten, 62, 291.

Gueben-Venière, S. (2011). How can mental maps, applied to the coast environment, help in collecting and analyzing spatial representations? EchoGéo, 17, Article 17. https://doi.org/10.4000/echogeo.12625.

Hauthal, E., \& Burghardt, D. (2019). Action, Emotion, Opinion - A Taxonomy of Human Reactions Expressed in Location-Based Social Media. Abstracts of the ICA, 1, 1-2. https://doi.org/10.5194/ica-abs-1-108-2019.

Huang, H., Gartner, G., Klettner, S., \& Schmidt, M. (2014). Considering Affective Responses towards Environments for Enhancing Location Based Services. ISPRS - International Archives of the Photogrammetry, Remote Sensing and Spatial Information Sciences, XL-4, 93-96. https://doi.org/10.5194/isprsarchives-XL-4-93-2014.

Lynch, K. (1960). The image of the city (Vol. 11). MIT press.

Milgram, S., \& Jodelet, D. (1976). Psychological Maps of Paris.

Nold, C. (2009). Emotional cartography: Technologies of the self. publisher not identified.

Panek, J., \& Benediktsson, K. (2017). Emotional mapping and its participatory potential: Opinions about cycling conditions in Reykjavík, Iceland. Cities, 61, 65-73. https://doi.org/10.1016/j.cities.2016.11.005.

Pearce, M. W. (2008). Framing the Days: Place and Narrative in Cartography. Cartography and Geographic Information Science, 35(1), 17-32. https://doi.org/10.1559/152304008783475661.

Reuchamps, M., Kavadias, D., \& Deschouwer, K. (2013). Drawing Belgium: Using Mental Maps to Measure Territorial Conflict. Territory, Politics, Governance, 2, 30-51. https://doi.org/10.1080/21622671.2013.814549. 